\title{
One-transistor one-resistor (1T1R) cell for large-area electronics
}

N. Ghenzi, M. Rozenberg, L. Pietrobon, R. Llopis, R. Gay, M. Beltrán, M. Knez, L. Hueso, and P. Stoliar

Citation: Appl. Phys. Lett. 113, 072108 (2018); doi: 10.1063/1.5040126

View online: https://doi.org/10.1063/1.5040126

View Table of Contents: http://aip.scitation.org/toc/apl/113/7

Published by the American Institute of Physics

\section{Articles you may be interested in}

Controlling the thin interfacial buffer layer for improving the reliability of the $\mathrm{Ta} / \mathrm{Ta}_{2} \mathrm{O}_{5} / \mathrm{Pt}$ resistive switching memory

Applied Physics Letters 113, 072902 (2018); 10.1063/1.5040430

Artificial electronic synapse characteristics of a Ta/Ta2 $\mathrm{O}_{5-x} / \mathrm{Al}_{2} \mathrm{O}_{3} / \mathrm{lnGaZnO} 4$ memristor device on flexible stainless steel substrate

Applied Physics Letters 113, 013503 (2018); 10.1063/1.5027776

Configurable ultra-low operating voltage resistive switching between bipolar and threshold behaviors for Ag/ $\mathrm{TaO}_{\mathrm{X}} / \mathrm{Pt}$ structures

Applied Physics Letters 113, 112103 (2018); 10.1063/1.5047925

Improvement of durability and switching speed by incorporating nanocrystals in the $\mathrm{HfO}_{\mathrm{x}}$ based resistive random access memory devices

Applied Physics Letters 113, 023105 (2018); 10.1063/1.5030780

A radiation-hardening $\mathrm{Ta} / \mathrm{Ta}_{2} \mathrm{O}_{5-\mathrm{x}} / \mathrm{Al}_{2} \mathrm{O}_{3} / \mathrm{InGaZnO}_{4}$ memristor for harsh electronics

Applied Physics Letters 113, 122907 (2018); 10.1063/1.5045649

Half-volt operation of IGZO thin-film transistors enabled by ultrathin $\mathrm{HfO}_{2}$ gate dielectric

Applied Physics Letters 113, 063501 (2018); 10.1063/1.5037410

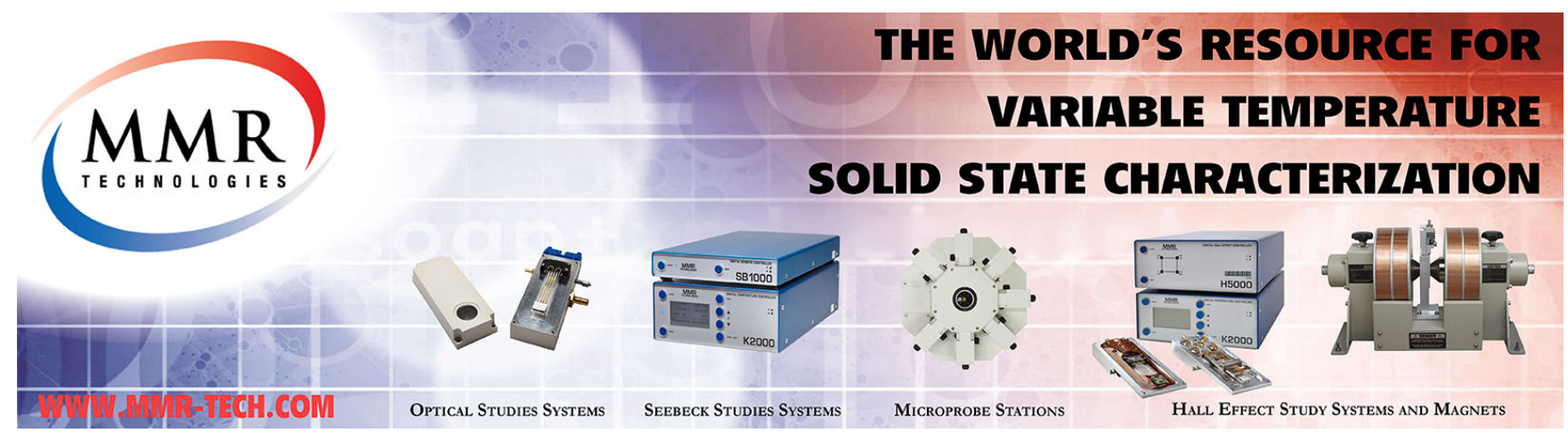




\title{
One-transistor one-resistor (1T1R) cell for large-area electronics
}

\author{
N. Ghenzi, ${ }^{1,2,3}$ M. Rozenberg, ${ }^{4}$ L. Pietrobon, ${ }^{1}$ R. Llopis, ${ }^{1}$ R. Gay, ${ }^{1}$ M. Beltrán, ${ }^{1}$ M. Knez,,${ }^{1,5}$ \\ L. Hueso, ${ }^{1,5}$ and P. Stoliar ${ }^{1,6, a)}$ \\ ${ }^{1}$ CIC nanoGUNE, 20018 Donostia-San Sebastian, Basque Country, Spain \\ ${ }^{2}$ Consejo Nacional de Investigaciones Científicas y Técnicas (CONICET), C1033AAJ Buenos Aires, Argentina \\ ${ }^{3}$ Facultad de Ingeniería y Agronomía, UCA, C1107AFF Buenos Aires, Argentina \\ ${ }^{4}$ LPS, UMR 8502, CNRS - Université Paris Sud, 91405 Orsay, France \\ ${ }^{5}$ IKERBASQUE, Basque Foundation for Science, 48011 Bilbao, Basque Country, Spain \\ ${ }^{6}$ National Institute of Advanced Industrial Science and Technology (AIST), 305-8565 Tsukuba, Japan
}

(Received 14 May 2018; accepted 25 July 2018; published online 14 August 2018)

\begin{abstract}
We developed a one-transistor one-resistor cell composed of one $\mathrm{TiO}_{2}$-based resistive switching (RS) device and one ZnO-based thin-film transistor (TFT). We study the electric characteristics of each component individually, and their interplay when both work together. We explored the direct control of bipolar RS devices, using our TFTs to drive current in both directions. We also report striking power implications when we swap the terminals of the RS device. The target of our work is the introduction of RS devices in large-area electronic (LAE) circuits. In this context, RS devices can be beneficial regarding functionality and energy consumption, when compared to other ways to introduce memory cells in LAE circuits. Published by AIP Publishing. https://doi.org/10.1063/1.5040126
\end{abstract}

Resistive switching (RS) devices are simple capacitorlike two-terminal structures in which the resistance can be adjusted by electric pulsing. ${ }^{1-4}$ RS devices are attracting much attention as high capacity memories and in the field of neuromorphic systems because they can be integrated into high-density crossbar arrays. ${ }^{5-8}$ It is clear that large-area electronics (LAE, e.g., flexible and transparent electronics) is not the technology to implement large neuromorphic circuits or high-density memories. Nevertheless, the introduction of RS devices can be very beneficial for LAE because, in some cases, it can help to reduce the number of components. Also, RS devices are nonvolatile, which is good for power saving.

As an example, let us consider that we have to integrate a few bits of memory in a flexible circuit, which are generally made with thin-film transistors (TFTs). We should base the memory cells on one of the following technologies: dynamic or static random-access memory (DRAM, SRAM), floating-gate (FG) transistors, or RS devices. SRAM typically requires 6 TFTs per bit and is volatile. DRAM cells are simple, but they need a complicated refresh circuit. FG transistors are nonvolatile and compact, but the technology to make the floating gate is generally out of reach for the flexible electronics manufacturing plants.

RS devices are an attractive alternative; an RS-based memory cell requires only two components and is nonvolatile, simple to use and fabricate, and compatible with LAE fabrication processes. The two required components are one RS device and one selection device. In general, selection devices-an electronic component that controls the current flow through the RS device-are highly nonlinear components or transistors. ${ }^{9-11}$ In the latter case, the subcircuit composed of one RS device and one transistor is called a one-transistor one-resistor (1T1R) cell. ${ }^{12-15}$

In standard integrated circuits, the transistor is a singlecrystal MOS. Here, we study a 1T1R cell using a thin-film

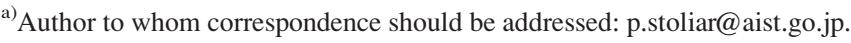

transistor (TFT) instead. ${ }^{16}$ This minor change has substantial implications. The performance of TFT is not equivalent to silicon transistors. Silicon transistors behave as ideal switches when controlling RS devices. On the contrary, the ON resistance of the channel in TFTs is comparable (if not higher) to the resistance of the RS device. As a general rule, TFT does not have any advantage over the single-crystal Si-based transistors regarding power consumption and switching performance. But in some cases, such as LAE, single-crystal MOS technology cannot be used; we are focusing on those cases.

Figure 1 presents our 1T1R cell composed of an n-type TFT driving a bipolar RS device. The nodes of this circuit are the transistor gate $(\mathrm{G})$, the source $(\mathrm{S})$, the drain connected to the bottom electrode of the RS device (D), and the top electrode $(\mathrm{T})$ of the RS device. The substrate is highly doped
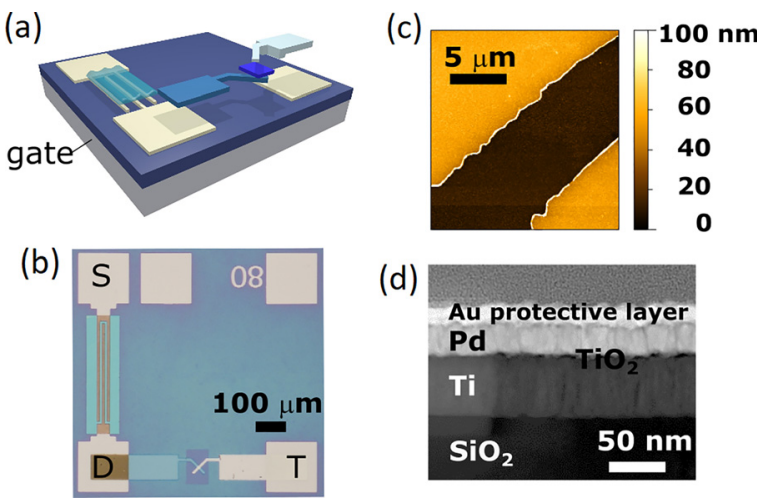

(d)

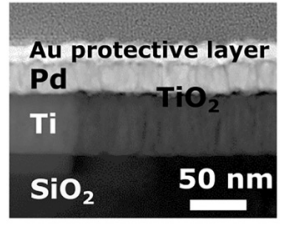

FIG. 1. (a) Exploded view of the structure, composed of a $\mathrm{ZnO}$ :N-based transistor and an amorphous $\mathrm{TiO}_{2-\mathrm{X}}$-based RS device. The substrate behaves as the gate electrode. The fabrication involves five photolithography steps. The first two steps define the electrodes and access pads (Au/Ti) and the semiconducting channel of the transistor $(\mathrm{ZnO}: \mathrm{N})$. The final three stages define the bottom electrode $(\mathrm{Ti})$, the active layer $\left(\mathrm{TiO}_{2-\mathrm{x}}\right)$, and the top electrode (Pd) of the RS device. (b) Optical image. There are three access pads, the source of the transistor (S), drain (D) connected to the bottom electrode of the RS device, and the top electrode of the RS device (T). (c) AFM close up of the transistor channel. (d) HRTEM image of the RS devices. 
$\mathrm{Si}^{++}$with a $300 \mathrm{~nm}$-thick thermal oxide layer, where the $\mathrm{Si}^{++}$ is the gate, and the $\mathrm{SiO}_{2}$ is the gate dielectric. The W/L is 80 (channel width $\mathrm{W}=800 \mu \mathrm{m}$ and channel length $\mathrm{L}=10 \mu \mathrm{m}$ ). The semiconducting channel, nitrogen-doped zinc oxide (ZnO:N), was deposited by Atomic Layer Deposition (ALD) at $95^{\circ} \mathrm{C}$. In addition to the zinc precursor (diethyl zinc, or DEZ) and $\mathrm{H}_{2} \mathrm{O}$, we used an ammonium hydroxide solution as a third precursor for nitrogen doping. We tested different doping levels looking for the highest ON/OFF ratio and the lowest leakage current. The best result was by alternating a pulse of $\mathrm{H}_{2} \mathrm{O}$ and another of a solution of $28 \% \mathrm{NH}_{3}$ in $\mathrm{H}_{2} \mathrm{O}$, after every DEZ pulse. $^{17}$

The RS device is a cross-shaped structure with an overlapping area of $5 \times 5 \mu \mathrm{m}^{2}$. The bottom electrode is made of Ti and the top electrode of $\mathrm{Pd}$. We deposited the $\mathrm{TiO}_{2-\mathrm{x}}$ layer by reactive sputtering at room temperature. We adjusted the oxygen content to have a forming-free operation, as reported elsewhere. ${ }^{18} \mathrm{~A}$ forming-free RS device does not need any electrical preconditioning (electroforming) before use. Swapping the electrodes of the RS device is central to our study, i.e., whether we connect the Pd or the Ti electrode to the transistor. We called "direct" configuration when the Ti bottom electrode is connected to the drain electrode and "inverse" configuration when the Pd top electrode is connected to the transistor. See supplementary material for details on the fabrication. Overall, the maximum required temperature in all the fabrication processes is $95^{\circ} \mathrm{C}$ (ALD), which renders all our processes well compatible with LAE fabrication plants.

In this manuscript, we will first report the characterisation of the transistor and the RS device separately and then the study of both devices working together. We start with the transistor. We used two source measure units (SMU): one biased the gate by applying $\mathrm{V}_{\mathrm{GS}}$ between $\mathrm{S}$ and the gate and the other SMU biased the channel between $\mathrm{S}$ and $\mathrm{D}\left(\mathrm{V}_{\mathrm{DS}}\right)$ and measured the current $\left(\mathrm{I}_{\mathrm{D}}\right)$.

Figure 2(a) presents the transfer characteristic of our n-type transistors. The $\mathrm{ZnO}: \mathrm{N}$ layer is near the subthreshold region at zero gate voltage $\left(\mathrm{V}_{\mathrm{GS}}\right)$ due to the nitrogen doping, which compensates most of the free electrons present in the $\mathrm{ZnO}$ film. Electrons are electrostatically injected into the $\mathrm{ZnO}: \mathrm{N}$ film as gate voltage increases. The transistor turns on when $\mathrm{V}_{\mathrm{GS}}$ exceeds the threshold voltage $\mathrm{V}_{\mathrm{TH}}=-1.5 \mathrm{~V}$. Electrons form a conducting channel in the $\mathrm{ZnO}: \mathrm{N}$ layer, with field-effect mobility (saturation regime) $\mu_{\mathrm{FET}}=2.5 \mathrm{~cm}^{2} /$ Vs (see supplementary material).

Figure 2(b) presents the output characteristics. We observe a strong asymmetry between positive and negative $\mathrm{V}_{\mathrm{DS}}$. In general, the output characteristic of n-type transistors is reported only for positive channel bias voltage $\left(\mathrm{V}_{\mathrm{DS}}\right)$. But in this work, we operate the transistor with positive and negative $\mathrm{V}_{\mathrm{DS}}$ because our resistive switching devices are bipolar.

We use the fundamental equations of field-effect transistors to explain the asymmetry in the output characteristics. We only study the linear regime $\left(\mathrm{V}_{\mathrm{GS}} \ll \mathrm{V}_{\mathrm{TH}}-\mathrm{V}_{\mathrm{DS}}\right)$. Based on the gradual channel model for thin-film transistors, ${ }^{19}$ the current through the channel is ${ }^{20}$

$$
I_{D}=\frac{W}{L} C_{i} \mu_{F E T} \int_{0}^{V_{D S}} V_{G S}-V_{T H}-v d v .
$$
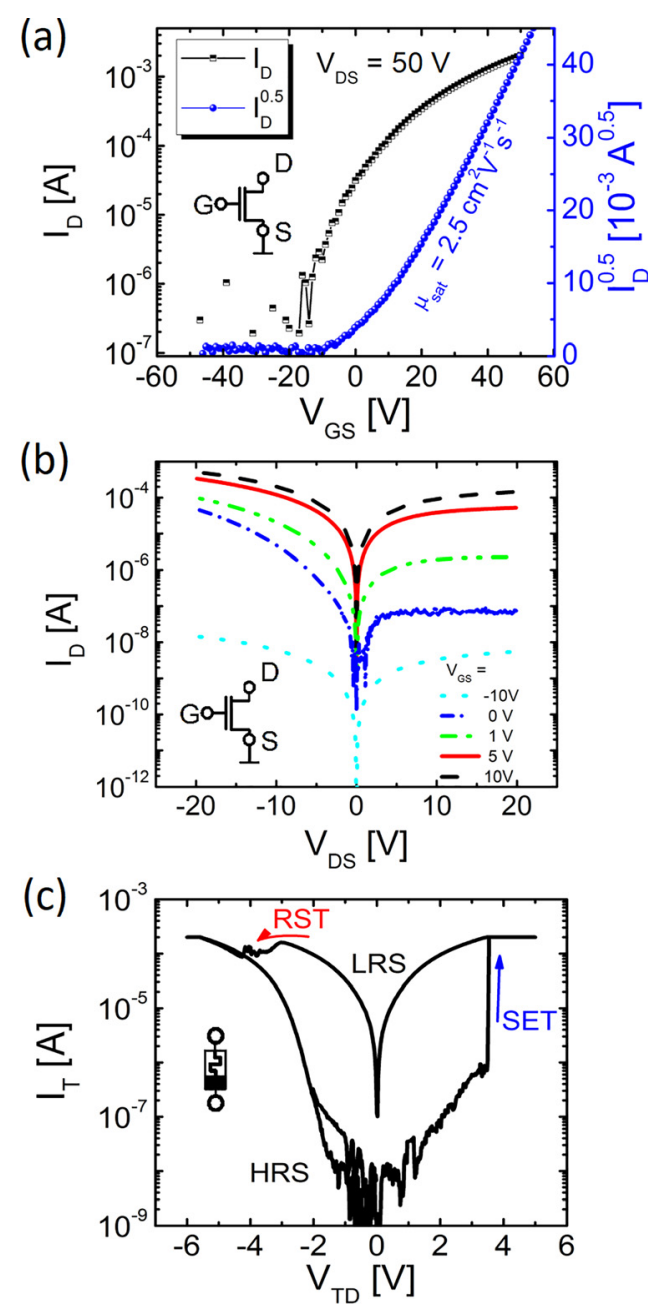

FIG. 2. (a) Transistor transfer characteristic ( $I_{D} v V_{G S}$ for $\left.V_{D S}=50 \mathrm{~V}\right)$. (b) Transistor output characteristics ( $I_{D}$ vs $V_{D S}$ ) measured with positive and negative channel bias. The strong asymmetry is central to our work. (c) I-V characteristics of the RS device.

Integrating, we obtain the gate-dependent resistance of the channel, $\mathrm{V}_{\mathrm{DS}} / \mathrm{I}_{\mathrm{D}}$

$$
R_{c h}=R_{c h}^{0} \frac{\left(V_{G S}-V_{T H}\right)}{\left(V_{G S}-V_{T H}\right)-\frac{V_{D S}}{2}},
$$

where $R_{c h}^{0}=\left[\frac{W}{L} C_{i} \mu_{F E T}\left(V_{G S}-V_{T H}\right)\right]^{-1}$. The asymmetry of the output characteristics is due to the term $\mathrm{V}_{\mathrm{DS}} / 2$. It is common practice to neglect this term, which leads to the standard linear regime formula $I_{D}=\frac{W}{L} C_{i} \mu_{F E T}\left(V_{G S}-V_{T H}\right) V_{D S} \cdot{ }^{21}$

The differential output conductivity $\mathrm{dI}_{\mathrm{D}} / \mathrm{dV}_{\mathrm{DS}}$ for positive $\mathrm{V}_{\mathrm{DS}}$ approaching $\mathrm{V}_{\mathrm{GS}}$ is

$$
\left.\frac{d I_{D}}{d V_{D S}}\right|_{V_{D S} \rightarrow V_{G S}}=0
$$

whereas for negative $V_{D S}$, it is

$$
\left.\frac{d I_{D}}{d V_{D S}}\right|_{V_{D S} \rightarrow-V_{G S}}=\frac{3}{2} \frac{1}{\left.R_{c h}\right|_{V_{D S} \rightarrow-V_{G S}}} \approx 30 \mathrm{usA} / \mathrm{V} \text { at } \mathrm{VGS}=5 \mathrm{~V}
$$

The difference in $\mathrm{dI}_{\mathrm{D}} / \mathrm{dV}_{\mathrm{DS}}$ is because the transistor current saturates for positive $\mathrm{V}_{\mathrm{DS}}$ (i.e., as the transistor 
approaches the saturation regime, the drain current becomes independent of the channel bias), but it always keeps increasing as the negative $\mathrm{V}_{\mathrm{DS}}$ gets higher (the drain current is proportional to the channel bias). We will see that this difference is essential for our work.

Next, we characterise the RS devices by connecting an SMU between pads D and T. Figure 2(c) shows the principal feature of our RS devices, an I-V loop with two branches, corresponding to the high-resistance state (HRS) and the low-resistance state (LRS). The transition from HRS to LRS, or SET, occurs when the voltage exceeds $4 \mathrm{~V}$. Reset (RST) happens at negative $\mathrm{V}_{\mathrm{TD}}<-3 \mathrm{~V}$. The ON/OFF ratio at low bias exceeds two orders of magnitude (HRS $=9.2 \times 10^{5} \Omega$ and $\mathrm{LRS}=6 \times 10^{3} \Omega$ ). This kind of resistive switching behaviour is a strong indication of the underlying physical mechanism. Strong electric fields drift oxygen vacancies back and forth from the $\mathrm{Pd} / \mathrm{TiO}_{2}$ interface, which modulates the resistance of the device. ${ }^{18}$

We now study the behaviour of both devices together. We used two source measure units. One SMU sets $\mathrm{V}_{\mathrm{GS}}$. The other SMU was connected between $\mathrm{S}$ and $\mathrm{T}\left(\mathrm{V}_{\mathrm{TS}}\right)$. It biased both the transistor channel and the RS device and measures the current $\left(\mathrm{I}_{\mathrm{T}}\right)$. We did not connect the $\mathrm{D}$ terminal. Regarding the SET and RST thresholds, one can define thresholds on $\mathrm{V}_{\mathrm{GS}}$ and $\mathrm{V}_{\mathrm{TS}}$ because the switching of the RS depends on both variables. Throughout this work, we concentrate on the thresholds on $\mathrm{V}_{\mathrm{TS}}$, for a fixed $\mathrm{V}_{\mathrm{GS}}$.

Figure 3(a) presents the $I-V$ characteristics of the "direct" configuration. We fixed $\mathrm{V}_{\mathrm{GS}}$ and swept $\mathrm{V}_{\mathrm{TS}}$. When the transistor is $\mathrm{OFF}\left(\mathrm{V}_{\mathrm{GS}}=-10 \mathrm{~V}\right)$, the current is due to its leakage current; $I_{T}=10^{-10} \mathrm{~A}$. When the transistor is activated $\left(\mathrm{V}_{\mathrm{GS}}=+10 \mathrm{~V}\right)$, we observe the typical switching response with two resistance states. RST was at $-3 \mathrm{~V}$ for the individual RS device, but now, it occurs at $\mathrm{V}_{\mathrm{TS}}=-4 \mathrm{~V}$. This

(a)

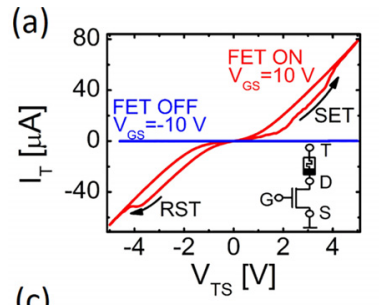

(b)

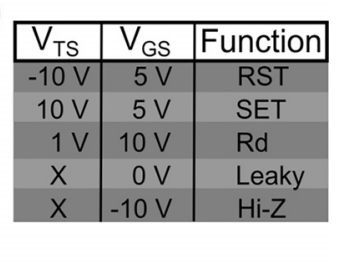

(c)

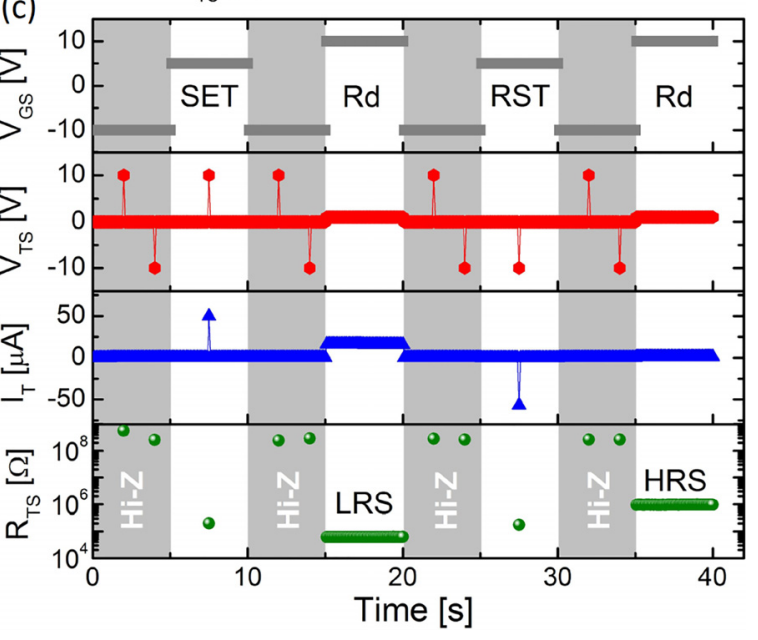

FIG. 3. (a) Electrical response in the "direct" configuration. (b) Table of the different operational modes. (c) Typical operational sequence. difference is because a significant fraction of $\mathrm{V}_{\mathrm{TS}}$ drops at the transistor. The SET remains at $4 \mathrm{~V}$. The current is around $50 \mu \mathrm{A}$ in both SET and RST.

Figure 3(b) presents the conditions for writing and reading information when operating with voltage pulses. We perform the writing functions (SET and RST) at $\mathrm{V}_{\mathrm{GS}}=5 \mathrm{~V}$ and $\mathrm{V}_{\mathrm{TS}}=+/-10 \mathrm{~V}$. One would expect that $\mathrm{V}_{\mathrm{GS}}=0 \mathrm{~V}$ turns the transistor off and isolates the RS device, but actually, it leads to what we call "Leaky" state ( $\mathrm{V}_{\mathrm{GS}}$ is very close to $\left.\mathrm{V}_{\mathrm{TH}}\right)$. We biased the transistor with a negative $\mathrm{V}_{\mathrm{GS}}=-10 \mathrm{~V}$ to obtain a high impedance state (Hi-Z). The Hi-Z state is useful for energy saving and operation in matrices.

Figure 3(c) shows a sequence of reading/writing/idle operations. $\mathrm{V}_{\mathrm{GS}}$ and $\mathrm{V}_{\mathrm{TS}}$ panels present the voltage setting of the SMUs, while $\mathrm{I}_{\mathrm{T}}$ and $\mathrm{R}_{\mathrm{TS}}$ are actual measurements. The system was in the high-impedance (Hi-Z) state between operations. During the Hi-Z states, we applied $\mathrm{V}_{\mathrm{TS}}$ test pulses to verify the isolation.

We then tested if the "inverse" configuration is better than the "direct" configuration regarding energy consumption. Figure 4 presents the most revealing experiment. Initially, we tried to repeat the I-V curves of Fig. 3(a) $\left(\mathrm{V}_{\mathrm{GS}}=5 \mathrm{~V}\right)$, but we were not able to reset the device. We then repeated the scans, by increasing $\mathrm{V}_{\mathrm{GS}}$ in steps. The RST process happened only when the gate voltage was increased up to $40 \mathrm{~V}$, at $\mathrm{V}_{\mathrm{TS}}=19 \mathrm{~V}$ and $\mathrm{I}_{\mathrm{T}}=300 \mu \mathrm{A}$. The contrast between the two configurations is quite telling. While in the "direct" configuration the system operated with $10 \mathrm{~V} / 50 \mu \mathrm{A}$, it required $19 \mathrm{~V} / 300 \mu \mathrm{A}$ after swapping the pads of the RS device. Quite significantly, by choosing the right configuration ("direct" configuration), the gate bias and power $\left(\mathrm{V}_{\mathrm{TS}}\right.$ $\times \mathrm{I}_{\mathrm{T}}$ ) requirements can be reduced by approximately one order of magnitude (relative to the "inverse" configuration).

Figure 5 helps us to rationalise the difference between both configurations. It presents the operational conditions of the transistor during SET and RST as load-line plots, which are common in the analysis of nonlinear circuits.

During SET, the load resistance of the transistor (i.e., the RS device) largely decreases (it switches from HRS to LRS). In the "direct" configuration, the operating point enters in the saturation regime of the transistor, where it limits the current. Here, we have a crucial difference between both configurations. In the "inverse" configuration, there is a runaway process during the SET. The current largely increases due to the

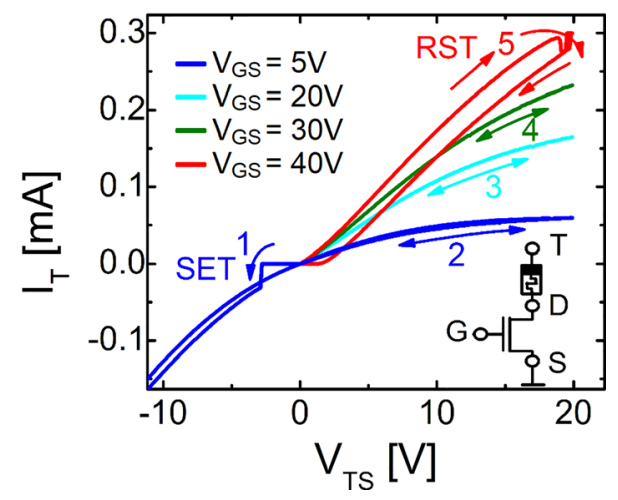

FIG. 4. "Inverse" configuration. In this configuration, we had to increase the gate voltage to $40 \mathrm{~V}$ and the channel bias to $20 \mathrm{~V}$ to induce the RST. 

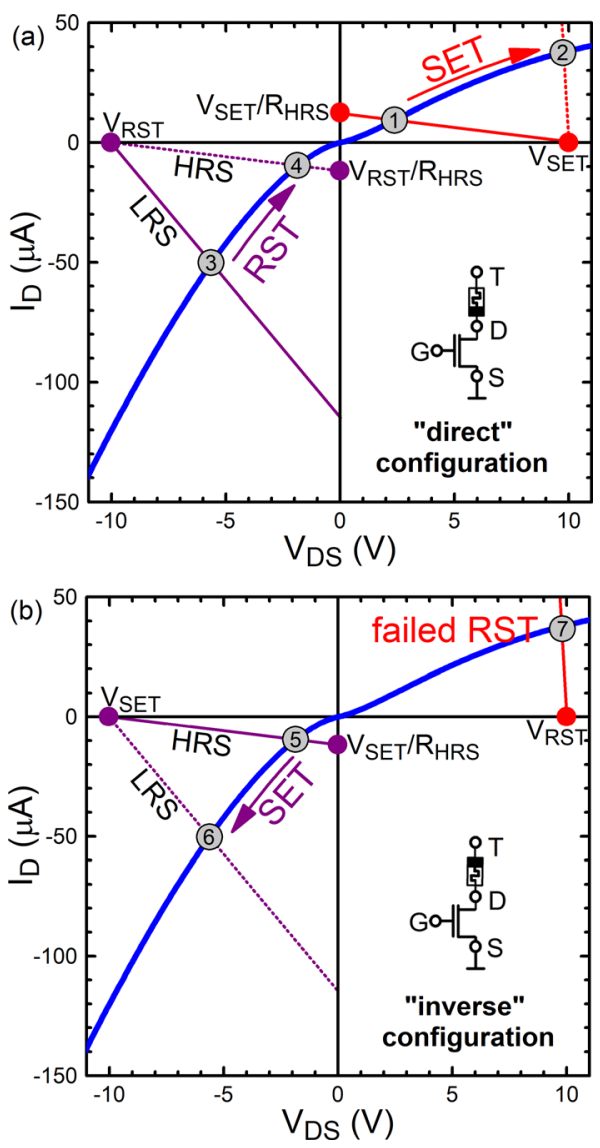

FIG. 5. Load line plots. The operating points are defined in a similar way to the load line analysis in conventional transistors. We used the output characteristics of the transistors $\left(I_{D}\right.$ vs. $\left.V_{D S}\right)$ for $V_{G S}=5 V$. For simplicity, we assumed linear LRS and HRS. $\mathrm{V}_{\mathrm{SET}}$ and $\mathrm{V}_{\mathrm{RST}}$ define points in the $\mathrm{V}_{\mathrm{DS}}$ axis (in red and purple). The HRS defines a point in the $\mathrm{I}_{\mathrm{D}}$ axis as $\mathrm{V}_{\mathrm{RST}}\left(\mathrm{V}_{\mathrm{SET}}\right)$ / $\mathrm{R}_{\mathrm{HRS}}$ (in red and purple). The load lines for the HRS connect these points, defining the operating points (points 1,4 , and 5). The LRS is highly nonlinear; therefore, to draw the load lines for the LRS, we fixed the operating points at $\left|\mathrm{I}_{\mathrm{D}}\right|=50 \mu \mathrm{A}$ (see the text, points 2,3 , and 6).

nonzero $\mathrm{dI}_{\mathrm{D}} / \mathrm{dV}_{\mathrm{DS}}$, while the load resistance gets continuously decreased. This process only stops after reaching a strong LRS that is very difficult to reset. ${ }^{22}$ By "strong LRS," we mean LRS with a very small resistance, much smaller than the ON channel resistance of the transistor. Therefore, practically all $\mathrm{V}_{\mathrm{TS}}$ drops on the transistor, and then, it is not possible to establish an electric field in the RS device to reset it. Another advantage of the "direct" configuration is that the current limitation avoids overshoots of current during the SET transition, which increases the endurance of the devices. $^{23}$

During RST, the operating point moves towards the origin because $V_{D S}$ decreases as the resistance of the RS devices increases much above $\mathrm{R}_{\mathrm{ch}}$. Therefore, the RST process does not require a limitation. However, the channel resistance should be as small as possible at the beginning of the RST, ideally, smaller than the LRS (otherwise, most $\mathrm{V}_{\mathrm{TS}}$ will drop on the transistor channel). We indeed have the lowest possible channel resistance during RST in the "direct" configuration. On the contrary, in the "inverse" configuration, the low $\mathrm{dI}_{\mathrm{D}} / \mathrm{dV}_{\mathrm{DS}}$ limits the available power, making harder to come back from a "strong LRS."
The transistor is in the linear regime $\left(\mathrm{V}_{\mathrm{GS}}=10 \mathrm{~V}, \mathrm{~V}_{\mathrm{DS}}\right.$ $<\mathrm{V}_{\mathrm{TS}}=1 \mathrm{~V}$ ) during the "read" operation ( $\left.\mathrm{Rd}\right)$.

In conclusion, we reported a 1T1R cell where a TFT controls a bipolar resistive switching device. We studied the use of TFTs driving current in both directions. We also tested the effect of swapping the terminals of the RS device and discussed its implications regarding power requirements. A practical pulsing protocol to operate in a logic circuit was defined. The target of our work is the development and implementation of a building block that can ease the introduction of RS devices in large-area electronic circuits. It is useful in the cases where conventional single-crystal Si transistors cannot be used.

See supplementary material for details on fabrication, extraction of the FET parameters, and characterization of the RS devices.

P.S. acknowledges the support of the Spanish Ministry of Economy through the Ramón y Cajal (No. RYC-201201031).

${ }^{1}$ M. J. Rozenberg, Scholarpedia 6(4), 11414 (2011).

${ }^{2}$ D. S. Jeong, K. M. Kim, S. Kim, B. J. Choi, and C. S. Hwang, Adv. Electron. Mater. 2, 1600090 (2016).

${ }^{3}$ J. S. Meena, S. M. Sze, U. Chand, and T.-Y. Tseng, Nanoscale Res. Lett. 9, 526 (2014).

${ }^{4}$ Y. Hu, S. Zhang, X. Miao, L. Su, F. Bai, T. Qiu, J. Liu, and G. Yuan, Adv. Mater. Interfaces 4, 1700131 (2017).

${ }^{5}$ K.-H. Kim, S. Gaba, D. Wheeler, J. M. Cruz-Albrecht, T. Hussain, N. Srinivasa, and W. Lu, Nano Lett. 12, 389-395 (2012).

${ }^{6}$ M. Prezioso, F. M. Bayat, B. D. Hoskins, G. C. Adam, K. K. Likharev, and D. B. Strukov, Nature 521, 61-64 (2015).

${ }^{7}$ G. C. Adam, B. Chrakrabarti, H. Nili, B. Hoskins, M. A. Lastras-Montaño, A. Madhavan, M. Payvand, A. Ghofrani, K.-T. Cheng, L. Theogarajan, and D. B. Strukov, in 2017 IEEE 17th International Conference on Nanotechnology (IEEE-NANO), Pittsburgh, PA (2017), pp. 844-849.

${ }^{8}$ C. Li, M. Hu, Y. Li, H. Jiang, N. Ge, E. Montgomery, J. Zhang, W. Song, N. Dávila, C. E. Graves, Z. Li, J. P. Strachan, P. Lin, Z. Wang, M. Barnell, Q. Wu, R. S. Williams, J. J. Yang, and Q. Xia, Nat. Electron. 1, 52 (2018). ${ }^{9}$ J. Y. Seok, S. J. Song, J. H. Yoon, K. J. Yoon, T. H. Park, D. E. Kwon, H. Lim, G. H. Kim, D. S. Jeong, and C. S. Hwang, Adv. Funct. Mater. 24, 5316 (2014).

${ }^{10}$ K. J. Yoon, W. Bae, D.-K. Jeong, and C. S. Hwang, Adv. Electron. Mater. 2, 1600326 (2016).

${ }^{11}$ A. Chen, IEEE Trans. Electron Devices 62(9), 2857-2864 (2015).

${ }^{12} \mathrm{See}$ http://www.semicon.panasonic.co.jp/en/products/microcomputers/ mn1011/ for an example of a commercial microcontroller using RS devices.

${ }^{13}$ X. P. Wang, Z. Fang, X. Li, B. Chen, B. Gao, J. F. Kang, Z. X. Chen, A. Kamath, N. S. Shen, N. Singh, G. Q. Lo, and D. L. Kwong, in 2012 International Electron Devices Meeting (2012), pp. 20.6.1-20.6.4.

${ }^{14}$ H. Liu, H. Lv, B. Yang, X. Xu, R. Liu, Q. Liu, and S. Long, IEEE Electron Device Lett. 35, 1224-1226 (2014).

${ }^{15}$ S. Z. Rahaman, H.-Y. Lee, Y.-S. Chen, Y.-D. Lin, P.-S. Chen, W.-S. Chen, and P.-H. Wang, Appl. Phys. Lett. 110, 213501 (2017).

${ }^{16}$ C.-C. Chang, P.-T. Liu, C.-Y. Chien, and Y.-S. Fan, Appl. Phys. Lett. 112, 172101 (2018).

${ }^{17}$ Y. D. Su, W. C. Shih, and J. Y. Lee, Appl. Phys. Lett. 91, 122902 (2007).

${ }^{18}$ N. Ghenzi, M. J. Rozenberg, R. Llopis, P. Levy, L. E. Hueso, and P. Stoliar, Appl. Phys. Lett. 106, 123509 (2015).

${ }^{19}$ M. Shur, M. Hack, and J. G. Shaw, J. Appl. Phys. 66, 3371-3380 (1989).

${ }^{20}$ A. R. Brown, C. P. Jarrett, D. M. De Leeuw, and M. Matters, Synth. Met. 88, 37-55 (1997).

${ }^{21}$ M. C. Hamilton, S. Martin, and J. Kanicki, Chem. Mater. 16, 4699-4704 (2004).

${ }^{22}$ K. J. Yoon, S. J. Song, J. Y. Seok, J. H. Yoon, G. H. Kim, J. H. Lee, and C. S. Hwang, Nanotechnology 24, 145201 (2013).

${ }^{23}$ J. J. Yang, I. H. Inoue, T. Mikolajick, and C. S. Hwang, MRS Bull. 37, 131 (2012). 